Gdańsk 2019, Nr. 40

https://doi.org/10.26881/sgg.2019.40.17

Jadwiga Kita-Huber

Jagiellonen Universität Krakau / Uniwersytet Jagielloński w Krakowie

https://orcid.org/0000-0003-1406-4550

\title{
Der (un)menschliche Wissenschaftler. Jean Pauls Dr. Katzenbergers Badereise (1809) und die Frage nach dem Menschen
}

\begin{abstract}
In seiner späten Erzählung Dr. Katzenbergers Badereise konstruiert Jean Paul die Figur eines zynisch-nüchternen und von seinem Sujet besessenen Wissenschaftlers, der - ergriffen von besonderer Liebe für Abweichungen, Anomalien und Querulantentum - stets Grenzen überschreitet. Das Werk entstand in Auseinandersetzung mit zeitgenössischen naturwissenschaftlichen Streitfragen und anthropologischen Entwürfen und wurde sowohl von Schriftstellern (Goethe, E. T. A. Hoffmann, Tieck, Platen, J. Grimm) als auch Naturwissenschaftlern stark rezipiert. Der Anatomie-Professor Johann Friedrich Meckel widmete Jean Paul - als Reaktion auf dieses Werk - die Abhandlung De duplicitate monstrosa und bedankte sich in seinem Widmungsbrief explizit für Dr. Katzenberger. Im Beitrag soll geprüft werden, inwieweit Jean Pauls Text die Frage nach dem (Un-) Menschlichen im Menschen problematisiert und so das anthropologische Wissen seiner Epoche erweitert.

Schlüsselwörter: Jean Paul, späte Erzählungen, Naturwissenschaften, anthropologische Konzepte um 1800

The (un)human scientist. Dr Katzenbergers Badereise (1809) and the question of man. In his late story, Dr Katzenbergers Badereise Jean Paul sketches a figure of a modern scientist who is cynical, rational and possessed by his work. He permanently transcends various barriers by having a particular liking to deviations, anomalies and strange behaviour. The story emerged amidst the fruitful discussion with contemporary scientific disputes and anthropological concepts hence it was recognized by writers (Goethe, E. T. A. Hoffmann, Tieck, Platen, J. Grimm) as well as by scientists. Johann Friedrich Meckel, a professor of anatomy, dedicated to Jean Paul his treatise De duplicitate monstrosa, where he expressed his appreciation for Dr Katzenberger. The paper will examine in what sense Jean Paul's text reflects the question of (un)humanity in a human being and the extent to which it contributed to the anthropological knowledge of the period.
\end{abstract}

Keywords: Jean Paul, late stories, natural sciences, anthropological concepts at the turn of the $19^{\text {th }}$ century

\section{Einleitung}

Der Hallenser Anatomie-Professor Johann Friedrich Meckel [der Jüngere] (1781-1833), einer der bedeutendsten Mediziner seiner Zeit, stellt seiner wissenschaftlichen Abhandlung De duplicitate monstrosa (Halle/Berlin 1815) eine Widmung für Jean Paul voran und bedankt 
sich im auf Latein verfassten Widmungsbrief explizit für dessen Erzählung Dr. Katzenberger Badereise. Er spricht den Dichter vertraulich mit einem „Du“ direkt an:

Wundere dich nicht, hochberühmter Mann, dass dein Name diesem Werk über Zwillingsmissgeburten vorangestellt wird [...]. Den Grund wirst du leicht erraten haben. Es ist die Geschichte Katzen bergers, des so eifrigen Liebhabers von Missgeburten, die von dir so kunstvoll erzählt ist, dass vielleicht in keiner Geschichte, die du uns geschenkt hast, deine Kenntnis des menschlichen Charakters eindringlicher und die Schärfe deines Geistes, durch die du dich auszeichnest, stärker hervorleuchtet. [...].“ (Meckel, zit. n. Häntzschel 2014: 132, Hrvg. i. O.)

Die Widmung verblüfte die Leser, die in der Erzählung vor allem ein Zerrbild des zeitgenössischen Naturforschers neuen Typus, eine unerbittliche Satire auf den Ärztestand sehen wollten, welche mit der Realität eher wenig zu tun hat (vgl. Häntzschel 2014: 133; Ueding 1993: 174). Auch noch zwei Jahrhunderte später wird die Verwandtschaft zwischen der fiktiven Figur Jean Pauls und dem realen Anatomen angezweifelt - meist in ethisch anmutenden Urteilen, Katzenberger präpariere aus Sensationslust, während Meckel den Ursachen der Abweichungen auf den Grund ging, um die Entstehung von Krankheiten zu erfassen (vgl. Noack 2013, Zugriffsdatum: 05.07.2018). Diese Abwehrhaltung gegen die Annahme des Realitätsbezugs resultiert vorerst aus der grotesk-abstrusen Zeichnung der Hauptfigur, die als Zyniker dem Vorbild klassischer Reinheit und Harmonie in mehrfacher Hinsicht Hohn spricht. Dabei gewinnt der Text gerade dank dieser Zeichnung einen neuen, eigenen Reiz und erlaubt Jean Paul etwas zu schildern, ohne dass er letztendlich eine klare wissenschaftliche oder moralische Position beziehen muss. Meckels Widmung liefert den Beweis, dass Jean Pauls Satire für zeitgenössische Mediziner Aufsehen erregend und aktuell war, dass er kein Gegenbild lieferte, das zeitgenössische Anatomen bekämpfen wollten. Im Gegenteil, Meckel nennt im angeführten Widmungsbrief sogar zwei Szenen, für die er sich bei Jean Paul wegen ihrer Relevanz für die Wissenschaft besonders bedanken möchte. Ich komme auf sie zum Schluss zu sprechen.

In Dr. Katzenbergers Badereise konstruiert Jean Paul die Figur eines zynisch-nüchternen und von seinem Sujet besessenen (Natur)Wissenschaftlers, der - ergriffen von der Liebe für Abweichungen und Anomalien - permanent Grenzen überschreitet, auch ,indem er alle kulturellen Überformungen aushebelt" (Pfotenhauer 2013: 334). Literatur- und wissensgeschichtlich steht die Figur an der Schwelle zwischen den verschiedenen, die Zeit um 1800 prägenden Modellen. Einerseits ist Katzenberger durch sein allgemeines Berufsprofil und die vertretenen wissenschaftlichen Theorien der Anthropologie der Aufklärung verpflichtet, wie zu zeigen sein wird, andererseits als ein Wesen dargestellt, das mit unbeherrschbaren Trieben konfrontiert wird und dämonische, teuflische Züge aufweist. Diese Triebe stehen nicht unvermittelt da, sondern lassen sich vor dem Hintergrund des Entwicklungsprozesses der modernen Spezialisierung und Ausdifferenzierung der Disziplinen erklären. ${ }^{1}$ Im Beitrag soll geprüft werden, inwieweit an der Figur Katzenbergers die Frage nach dem (Un-)Menschlichen problematisiert und so das anthropologische Wissen der Epoche erweitert wird. Welche neuen Dimensionen

\footnotetext{
1 Zu diesen Hintergründen vgl. Pott 2002: 163-178.
} 
des Menschlichen werden hier zur Schau gestellt? Wie verschiebt sich die Grenze bzw. wie wird auf Grund dieses Textes die Definition des (Un-)Menschlichen modifiziert?

\section{Zu einigen wissenschaftlichen Inspirationen Jean Pauls}

Wie viele andere Werke Jean Pauls entstand auch Dr. Katzenberger in enger Auseinandersetzung mit zeitgenössischen naturwissenschaftlichen Streitfragen und anthropologischen Entwürfen. Die Erzählung ist durchsetzt mit Namen und direkten Bezügen auf zeitgenössisch erkennbare Forscher, ihre Werke und Theorien - sowohl in den zahlreichen Digressionen und beigefügten so genannten,Werkchen' als auch auf der Ebene der Handlung in der eigentlichen Katzenberger-Geschichte. So erwirbt der Protagonist auf seiner Badereise das Standardwerk seiner Disziplin, Samuel Thomas Soemmerrings Abbildungen und Beschreibungen einiger Mißgeburten (1791), in dem bekanntlich nicht nur die Grenze des Menschen zum Tier, sondern auch zum Monströsen physiologisch bestimmt wird (vgl. Bergengruen/Borgards/Lehmann 2001:7-14). Bei der Erklärung gewisser Verhaltensweisen und Affekte der Figuren wird auf Albrecht von Hallers Nervenlehre und das neue Standardwerk der Physiologie Elementa physiologiae corporis humani (1784) zurückgegriffen. Der unmittelbare wissenschaftliche Kontext bleibt dabei durchgehend die Teratologie - die Lehre von körperlichen und organischen Fehlbildungen. Hier sind zum einen Bezüge auf konkrete Forscher, wie Philipp Friedrich Meckel [den Älteren] (1756-1803), den Begründer der wissenschaftlichen Teratologie, zum anderen auf wissenschaftliche Auffassungen über Missgeburten zu ermitteln, wie sie etwa in der zeitgenössisch geführten Debatte zwischen (mechanistischen) Präformationisten und (vitalistischen) Epigenetikern verhandelt wurden. ${ }^{2}$

\section{3. Über das Werk Dr. Katzenbergers Badereise}

Auf der Handlungsebene wird von einer mehrtägigen Reise „de [s] verwittibte[n] ausübende[n] Arzt [es] und anatomische[n] Professor [s] Katzenberger" $(\mathrm{I} / 6,89)^{3}$ zu dem Kurort Bad Maulbronn erzählt, zu der er seine einzige Tochter Theoda und - um die Kosten für die Kutsche zu sparen - den empfindsamen Dichter Theudobach von Nieß mitnimmt. Der eigentliche Zweck der Badereise ist jedoch nicht Katzenbergers Sorge um die Gesundheit seiner Familie,

2 Während die Präformationslehre von der Urzeugung ausgeht, d. h. den vorgeformten, seit Anfang der Schöpfung existierenden Keimen, in denen alles Leben angelegt ist, schlägt die Epigenese verschiedene Modelle einer ,eigenständigen Produktions- und Reproduktionskraft des Lebens'vor. Beide im 18. Jahrhundert miteinander konkurrierenden Theorien gehen auf mittelalterliche Spekulationen über die Art und Weise zurück, wie die Seele in den Leib eines Neugeborenen kommt (ob sie präformiert ist oder gerade erschaffen wird). Im Kontext der Missgeburten vgl. hierzu Wieland 2011:12-13.

3 Vgl.Jean Paul (2000): Dr. Katzenbergers Badereise nebst einer Auswahl verbesserter Werkchen. In: Ders.: Sämtliche Werke. Hg. von Norbert Miller. Abt. I, Bd. 6. Darmstadt: Wissenschaftliche Buchgesellschaft, 77-363. Diese Ausgabe wird im laufenden Text mit der Angabe der Abteilung, des Bandes und der Seitennummer, ohne Sigle, zitiert. 
sondern eine ,wissenschaftliche Angelegenheit, denn er hat vor, den Brunnenarzt Strykius ordentlich zu verprügeln. Dieser übte an seinen drei berühmten Meisterwerken - u. a. De monstris epistola - in Rezensionen und Antworten auf Katzenbergers gelehrte Antikritiken vielfach heftige Kritik. Die Bestrafung des Rezensenten gelingt durch eine List: Katzenberger verprügelt - scheinbar durch den Alkohol aus der Bahn geraten - den Gegner und zwingt ihn, seine Rezensionen zu widerrufen (vgl. Wieland 2008: 169). Diese Haupthandlung wird vom Erzähler mit zahlreichen Exkursen und beigefügten Extrablättchen unterbrochen, die inhaltlich keinerlei Bezug zur Geschichte aufweisen, so dass an Stelle eines geschlossenen Werks eine heterogene und disparate Zusammenstellung von Texten diverser Art vorliegt. Die Digressionen machen mehr als ein Drittel des Gesamtumfangs aus. Dieses ungewöhnliche Kompositionsprinzip wurde von der Forschung die „Korpus-Struktur“ genannt, was auf die im Werk zur Debatte stehende Textmetapher des Körpers und seiner Missbildungen zurückgeführt werden kann (vgl. Neumann 1975: 177). ${ }^{4}$

\section{Katzenberger als (un)menschlicher Wissenschaftler}

\section{1 Äußere Auffälligkeiten}

In der Erzählung wird in satirisch-grotesker Zuspitzung etwas Befremdendes und, wie der Dankes-Brief Meckels bewusst macht, damals noch Unausgesprochenes über den Wissenschaftler und allgemein über den Menschen vermittelt. Der Anatom Katzenberger wird als ein skrupelloser Forscher und fanatischer Monster-Sammler dargestellt. Er besitzt ein „Mißgeburten-Kabinett" (I/6, 93), das ihm die traditionelle Studierstube ersetzt, eine richtige Schatzsammlung aus raren Monstrositäten, wie sie im Zeitalter der sog. Naturalienkabinette und auch noch in der neuen wissenschaftlichen Ära sehr begehrt waren (vgl. Schmitz-Emans 2016: 107). Sein Charakter lässt sich als durch wissenschaftliche Kälte, fehlende Sensibilität und Egoismus geprägt beschreiben. Wie andere Arzt-Figuren Jean Pauls gibt er sich selbst ganz zugunsten seiner Karriere und seines Werks auf (vgl. Pott 2002: 163). Auch die Badekur dient nur der lange und rational durchgeplanten Rache an dem ungnädigen Rezensenten: „Denn dem Doktor wars schon im Leben bloß um die Wissenschaft zu tun, geschweige in der Wissenschaft selber." $(\mathrm{I} / 6,190)$ Was noch ansatzweise (mit)menschlich an ihm ist, wie die Mitnahme der Tochter zur Kur, erweist sich als „Abfallprodukt eines wissenschaftlichen Interesses“ (Dietrich 2003: 223), seiner „Liebe zur Anatomie“ (I/6, 129), wie es im Werk mehrmals heißt. Aber nicht allein aufgrund seiner Forschungsinteressen, sondern auch durch sein Verhalten tritt Katzenberger in der Erzählung als ein Sonderling auf, insofern er sich zu den herrschenden Sitten und Normen quer verhält bzw. keine Manieren und keine Tabus kennt. Sich zuweilen wünschend, selber ein „verzerrter Flügelmann und monströses Muster“ (I/6, 199) zu sein, kommt er in der Gesellschaft „als soziales Monster, als Störenfried der öffentlichen Ordnung“ (Wieland 2011: 10) an. Exzessive Beispiele liefert hierzu sein Essverhalten: Der Professor verzehrt Maikäfer und Spinnen, die er sich, frisch aus dem Keller geholt, aufs Brot streicht.

\footnotetext{
4 Zum Katzenberger-Korpus als Modell der Wek(chen)organisation vgl. rezent Kaminski 2017: 29-70.
} 
Da für ihn der Ekel die stärkste aller Empfindungen ist, erzählt er an der öffentlichen Tafel, er setze seinen Gästen gelegentlich „Ausgesuchtes“ vor, z. B. Schnepfendreck „wie gewöhnlich mit Butter auf Semmelscheiben geröstet" $(\mathrm{I} / 6,277) .^{5}$

\subsection{Liebe zu Missgeburten}

Zentral für die Figur Katzenbergers ist der bereits erwähnte Diskurs der Teratologie, der Missgeburten-Lehre. Seine Liebe zu Missbildungen und sein rücksichtsloses Begehren nach zugehörigen Objekten wird während der Badereise mehrmals unter Beweis gestellt. ${ }^{6}$ Zunächst in Szenen, in denen er mit obsessiver Hartnäckigkeit, aber methodisch gesichert, um empirische Belege ringt. Hier wird er als Zyniker par excellence gezeigt, der die Welt und den Menschen auf das bloß Physische bzw. Materielle reduziert und alles Geistige ausblendet. So scheut er sich während einer Übernachtung nicht, auf einem nahgelegenen Friedhof „Knochen einzusammeln“ $(\mathrm{I} / 6,183)$ und das Gebeinhaus mit „ein[em] wohlerhaltene[n] Kindergerippe“ (I/6, 184) und „mehren Köpfen und Rückgraten behangen“ (I/6, 184f.) zu verlassen. Das Knochen-Sammeln war in dieser Zeit an sich zwar nichts Außergewöhnliches. Wie Monika Schmitz-Emans bemerkt, spielt „gerade das Sammeln von Knochen, insbesondere von Schädeln, [...] eine wichtige Rolle in der zeitgenössischen empirischen Anthropologie“ (Schmitz-Emans 2016: 109)7, sah man gerade im Schädel „den Menschen gleichsam konzentriert auf seine prägnantesten Merkmale vorliegen“ (ebd.). Aber Katzenberger geht für seine Abnormitäten-Sammlung buchstäblich über die Leichen, nichts als der Forschungsgegenstand setzt seinem Handeln Maßstäbe. Dabei verliert er nicht nur die letzten Anzeichen von Empathie, sondern wird auch bösartig und zynisch: So erschreckt er, glücklich über den Fund, seine Tochter Theoda und den Dichter Theudobach bei einem romantischen Mondscheinspaziergang, indem er ihnen einen Kinderschädel wie einen Kegel zurollt (I/6, 184). Wie Alexander Košenina zu Katzenbergers Tätigkeit bemerkt: Was an seiner Sammelei empört, ist

nicht die bloß vordergründig anstößig wirkende Spezialisierung auf Abnormalität [...], sondern die unendliche Zergliederung, Fragmentierung, Vereinzelung und Isolation. Sie steuern dem anthropologischen Erfolgsprogramm der Aufklärung, dem ganzen Menschen, zynisch entgegen. Organische Einheit, die im Sinne Goethes die mannigfaltigen Einzelheiten in der Idee einer lebendigen Ganzheit erscheinen läßt, wird so systematisch torpediert." (Košenina 2004, 193)

Die Ablösung vom anthropologischen Programm der Aufklärung, die hier in der ,unendlichen Zergliederung' zum Ausdruck kommt, ist für die Frage nach dem Menschlichen von gesteigerter Bedeutung. Thematisiert die Erzählung eigentlich, ob die hier beschriebene

5 Wie Wieland anmerkt: „Mit dem Verstoß gegen die Grenzen des guten Geschmacks auf der diegetischen Ebene verstößt die Erzählung insgesamt gegen die Regeln der klassischen Ästhetik, deren Ideale sich auch vom Gebot der Ekelvermeidung herleiten" (Wieland 2011: 12).

6 Zu Missgeburten bei Jean Paul vgl. Bergengruen 2003.

7 Schädel sammelten auch der niederländische Anatomieprofessor Petrus Camper und der Göttinger Naturgelehrte Johann Friedrich Blumenbach. Vgl. ebd. 
,Fragmentierung' und ,Vereinzelung' einen Einfluss auf die psychische Verfassung des Menschen haben könnte? Auf diese Frage komme ich im Schlussabsatz zu sprechen.

Ein weiteres Beispiel für Katzenbergers Liebe zu Missgeburten finden wir in der Szene, in der er Soemmerrings Beschreibungen einiger Mijgeburten (1791) gewinnt und gleichzeitig einem Stadt-Apotheker ein ungewöhnliches Monstrum, „einen gut ausgestopften, achtbeinigen Doppel-Hasen“ $(I / 6,129)$ stiehlt, da er die benötigte Geldsumme nicht aufbringen kann. Diese Handlungen werden von Aussagen begleitet, die sich im medizinhistorischen Kontext der Teratologie um 1800 bewegen, als die Missgeburt - die nicht mehr nur als Kuriosität bewundert wird - nun auch wissenschaftlich interessant wird. Katzenberger schwärmt wie folgt von Missbildungen:

Inzwischen habe ich [...] ohne Bedenken die allgemeine Gleichgültigkeit gegen echte Missgeburten gerügt und es sei frei herausgesagt, wie man Wesen vernachlässigt, die uns am ersten die organischen Baugesetze [...] durch ihre Abweichungen gotischer Bauart lehren können. Gerade die Weise, wie die Natur zufällige Durchkreuzungen und Aufgaben (z. B. zweier Leiber mit einem Kopfe) doch organisch aufzulösen weiß, dies belehrt. Sagen Sie mir nicht, dass Mißgeburten nicht bestehen, als widernatürlich; jede musste einmal natürlich sein, sonst hätte sie nicht bis zum Leben und Erscheinen bestanden;" $(I / 6,128)$

Der Doktor vertritt also die Meinung, dass es sich bei Monstren nicht um widernatürliche, sondern durchaus natürliche Erscheinungen handelt. Damit schließt er sich der modernen Teratologie und genau der Theorie der Epigenetiker an, die bemüht waren, „die organischen Deformationen in ein quasi-evolutionäres naturwissenschaftliches Erklärungsmodell einzubetten, anstatt die Missgeburt als unnatürlich auszugrenzen“" (Wieland 2011:12). An der „gotischen Bauart" (I/6, 128) der Natur könne man durch Abweichungen deren Baugesetze noch eingehender analysieren; gerade anhand der Abweichung lasse sich die Natur genauer verstehen.

Um seine hohe Einschätzung der Monstra seiner Tochter Theoda gegenüber glaubhaft zu machen, führt Katzenberger einen Beweis aus eigenem Leben an, als er seiner schwangeren Frau mit seinen „Kabinetts-Pretiosen“ Angst einjagte, mit der Hoffnung, sie würde sein Kabinett mit einem missgestalteten Kind bereichern:

ich könnte z. B. mit einer weiblichen Mißgeburt [...] in den Stand der Ehe treten; und ich will dirs nicht verstecken, Theoda - da die Sache aus reiner Wissenschaftsliebe geschah und ich gerade an der Epistel de monstris schrieb -, daß ich an deiner sel. Mutter während ihrer guten Hoffnung eben nicht sehr darauf dachte, aufrechte Tanzbären, Affen oder kleine Schrecken und meine Kabinetts-Pretiosen fern von ihr zu halten, weil sie doch im schlimmsten Falle bloß mit einem monströsen Ehesegen mein Kabinett um ein Stück bereichert hätte; aber leider, hätt' ich beinah' gesagt, aber gottlob sie bescherte mir dich als eine Bestätigung der Lavaterschen Bemerkung, daß die Mütter, die sich in der Schwangerschaft von Zerrgeburten am meisten gefürchtet, gewöhnlich die schönsten gebären. (I/6, 129)

Katzenbergers skrupellose wissenschaftliche Neugier macht also selbst vor dem Wohlbefinden der eigenen Familie nicht halt. Die heimliche Hoffnung, seine Frau möge ein Monstrum auf die Welt bringen, erfüllt sich nicht. In der hier angeführten Erklärung, die sich auf Lavaters Anthropologie für Aerzte und Weltweise (von 1772) stützt, klingt die von der Frühen Neuzeit geerbte und in der Anthropologie des gesamten 18. Jahrhunderts kontroverse Frage an, „ob Mißgeburten durch maternale Impressionen entstehen können“" (Košenina 2004: 192). 
Katzenberger zufolge werden Missbildungen von den gleichen Naturgesetzen wie alles andere, das sog. „Alltagvieh“ (I/6, 128), hervorgebracht. Ein Monstrum stellt keine unausgebildete, zurückgebliebene Stufe des Organismus dar, sondern ist ein komplexes und weit entwickeltes Gebilde - eine Auffassung, die für Jean Paul auch werkästhetische Implikationen hat. So hält Katzenberger in einer der von seinem Umfeld gefürchteten Tischreden Folgendes fest:

Eine Mißgeburt ist mir als Arzt eigentlich für die Wissenschaft das einzige Wesen von Geburt und Hoch- und Wohlgeboren; denn ich lerne mehr von ihm als vom wohlgeborensten Manne. Aus demselben Grunde ist mir ein Fötus in Spiritus lieber als ein langer Mann voll Spiritus; und Embryonengläser sind meine wahren Vergrößer-Gläser des Menschen. - Auch [...] in jedem von uns [...] sind einige Ansätze zu einem Monstrum, aber sie werden nicht reif. (I /6, 198)

Die provokativ formulierte Aussage über den „Fötus im Spiritus“ ist im Kontext wissenschaftsgeschichtlicher Zusammenhänge zu verstehen. Als Missgeburtenspezialist will Katzenberger erstaunlicher Weise doch ein allgemeines anthropologisches Wissen vermitteln; so sind ihm Embryonengläser wahre „Vergrößer-Gläser des Menschen“ oder „Sehröhre und Operngucker ins Lebensreich“ (I/6, 128). Mit diesen Mitteln bzw. Instrumenten will er nur noch genauer den Menschen studieren, ja sogar direkt ins „Lebensreich“ hineinschauen; die Objekte werden also nicht aus purer Sensationslust gesammelt, sondern sollen beim Erfassen des Menschen - wie auch immer - hilfreich sein.

Anatomische Deformationen sind für Katzenberger somit keine Defekte im gängigen organischen Wachstum. Wie Magnus Wieland bemerkt, „vielmehr erscheint ihm der gesunde Organismus gerade umgekehrt als Schwundstufe monströser Missbildungen, als Träger unterentwickelter Ansätze zur Missgeburt." (Wieland 2011: 13) Die Aussage, in jedem von uns seien Ansätze zu einem Monstrum, die sich im Text auf rein physische Verunstaltungen bezieht, ist auch im übertragenen Sinne zu verstehen - es mag sich um Ansätze zu einem Scheusal oder eben einem Unmenschen handeln.

Um die Nobilitierung der Monstra noch stärker zu unterstreichen, setzt Katzenberger die physische Anomalie der Missgeburt parallel zur Einmaligkeit des Genies, das sich ebenso aufgrund seiner Andersartigkeit bzw. Originalität von dem ,Normalen' (und der Masse) abhebt. Damit spitzt er seine Position noch einmal zu: „Wer kann [...] eine Mißgeburt, die sich so wenig als ein Genie fortpflanzt - denn sie ist selber ein körperliches, eine Einzigperle - nicht einmal ein Sonntagkind, sondern ein Schalttagkind -, ersetzen, ich bitte jeden?"“ (I/6, 128). Werden Genie und Monstrum im Hinblick auf ihre Originalität gleichgesetzt, so wird damit der herkömmliche Geniebegriff, wie ihn die klassische Ästhetik kennt, aufgelöst (vgl. Wieland 2011: 13).

\section{3 Die Apologie der Missgeburt und ihre Bedeutung für die Werkästhetik}

Die angeführte Aussage macht deutlich, dass Katzenbergers Missgeburten-Theorie auch poetologisch-ästhetische Konsequenzen hat. Wie bei anderen Schriftstellern um 1800, kommt es auch bei Jean Paul zu einem Konnex zwischen Anthropologie und Ästhetik 
(vgl. Bergengruen/Borgards/Lehmann 2001: 9); naturwissenschaftliche Konzepte werden auf poetologische Konzepte und die konkrete Schreibpraxis übertragen. Die angeführte Theorie der Epigenetiker, nach der genetische Veränderungen am menschlichen Organismus erst im Prozess seiner Entwicklung entstehen (und nicht schon im Embryo angelegt sind), dient ihm zur Etablierung eines unkonventionellen ästhetischen Verständnisses des Genies. Und wie Magnus Wieland festhält: „Dazu erfordert es auch gar keine grossen Umdeutungsversuche, denn selbst gewisse Teratologen attestierten der Missgeburt einen hohen ästhetischen Wert" (Wieland 2011: 13). Mit der Missgeburt wird eine Form dichterischen Werks bestimmt, die vom herrschenden „Ideal der Organizität“, eines organischen (gesunden) Kunstwerks, abweicht. Diese Form bedeutet ein noch komplexeres, höher entwickeltes Gebilde, das über einem vollkommenen, harmonisch gebauten Körper (Werk) stehen kann bzw. steht. Die Missgeburt wird, wie Magnus Wieland zunächst zeigte, zu Jean Pauls Chiffre im Rahmen einer „Ästhetik der Abweichung“( Wieland 2011: 15). Sie bezieht sich selbstreferenziell auf den digressiven Stil und die Komposition von Katzenberger, aber auch generell aufJean Pauls Textarchitektur, die der klassischen Vorstellung vom Werk als einem "in sich geschlossenen Organismus" widerstrebt (vgl. Helmreich 2002: 109). Die dem Katzenberger eingeschriebene Apologie des eigenen Werkkonzepts erscheint mir als besonders aufschlussreich, bedenkt man, welche Irritationen Jean Paul mit seinen Werken bei den berühmten Zeitgenossen Goethe und Schiller hervorgerufen hat. So schickte Goethe den im Vergleich zu Katzenberger doch viel klassischer komponierten Roman Hesperus an Schiller mit der Anmerkung weiter, es handle sich hierbei um einen „Tragelaph“, also im Verständnis der beiden Dichter um „ein mißgestaltetes oder groteskes Kunstwerk, [...] dem es [...] an innerer Harmonie und Ausgewogenheit seiner Teile [mangelt]" (Kemp/Miller/Philipp 1963: 14).

\section{Rezeption der Katzenberger-Geschichte bei den Zeitgenossen}

Der in Jean Pauls Erzählung präsentierte Typus des Wissenschaftlers und Sammlers findet im Laufe des 19. Jahrhunderts Gefallen bei Autoren, die gegen das idealistische Klassikbild opponieren oder ihm zumindest ferner stehen. Während E. T. A. Hoffmann in seiner Erzählungen-Sammlung Serapions-Brüder die Gestalt des Zynikers Katzenberger begrüßt, wird sie von Tieck, Platen oder Jacob Grimm missbilligt. ${ }^{8}$

Brisant ist auch die Wirkung der Erzählung gleich nach ihrem Erscheinen, wie schon die Differenzen zwischen der ersten (1808) und der zweiten (1822) Auflage zeigen, in die Jean Paul u. a. explizite Bezüge auf den eingangs erwähnten Johann Fr. Meckel einbaut. Wie erwähnt, bedankt sich Meckel in seiner Abhandlung besonders für zwei Episoden aus der Geschichte Katzenbergers:

8 „Während [manche] [...] Goethes Konzeption nahe bleiben, inszenieren andere Autoren sonderbare, abseitige, manische oder kriminelle und unheimliche Sammlerfiguren. Solcher Wandel erklärt sich nicht nur aus einem veränderten Bild vom Menschen, sondern auch aus ästhetischen und narrativen Erwägungen. Tatsächlich steckt ja spannungsreicheres literarisches Potential in Entwürfen skurriler oder grotesker Sammlerfiguren und damit in Texten, die keine identifizierende, zustimmende Lektüre erlauben, sondern eher provozieren [...]“ (Häntzschel 2014: 136 - meine Hrvg.). 
Nachdem du in dieser Geschichte zwei Dinge bestens dargelegt hast, erstens durch den Umriss des Kampfes mit dem Apotheker, wie ein tüchtiger Anatom selbst unter Lebensgefahr merkwürdige und seltene Objekte erwerben muss, sodann, durch die Bestrafung von Strykius, wie jene, die unter Missbrauch der Bezeichnung Kritiker die Tugend, die über sie gesetzt ist, zu beflecken und, wenn sie könnten, herabzuziehen streben, zu züchtigen sind, wird es wohl der Mühe wert sein, darzulegen, wie auch andere zu behandeln sind, die nicht auf nur eine einzige Weise den Namen eines Gelehrten besudeln. (Meckel, zit. n. Häntzschel 2014: 132-133)

Meckel nimmt also keinen Anstoß daran, dass ein Wissenschaftler seine Forschungsobjekte auch unter Lebensgefahr erwirbt und Tricks wie Diebstahl anwendet. Ganz im Gegenteil, er bewundert Katzenbergers Kompromisslosigkeit beim Sammeln und betont die Zweckrationalität eines solchen Handelns. Dabei musste das sog. allgemeine Lesepublikum „im Sammeln von Mißbildungen einen Affront gegen die positive Einschätzung des Sammelns in der klassischen und romantischen Literatur erkennen“" (Häntzschel 2014: 133) bzw. diese Tätigkeit auch moralisch verwerfen, bedenkt man, dass Katzenberger auch Friedhöfe aufsucht. Gutgeheißen wird von Meckel aber auch die an Strykius für sein Rezensionswerk vollzogene Prügel-Strafe; mehr noch, das wissenschaftliche Publikum soll sich an dieser Erzählung ein Beispiel nehmen, wie mit unwürdigen Rezensenten umzugehen ist.

\section{Fazit}

In Katzenberger integriert Jean Paul tatsächlich neue Bereiche in die Definition des Menschen, auch wenn er nicht wertet, was an seiner Figur menschlich und unmenschlich ist. Wie die meisten Anthropologen um 1800 bestimmt auch er den Menschen von seinen äußeren Grenzen her, und zwar durch die Einbeziehung der wissenschaftlichen Manie als eines Grenzfalls. Seine Erweiterung bzw. Grenzauflösung des Menschlichen korreliert mit seiner ästhetischen Theorie und Praxis. Die Problematisierung und Destabilisierung der äußeren Grenzen wird dabei vor dem Hintergrund der raschen Entwicklung der Naturwissenschaften zu Beginn des 19. Jahrhunderts vorgeführt. Katzenberger steht am Anfang einer Entwicklung, die angesichts der rasanten Spezialisierung und disziplinären Differenzierung das Allgemeine zunehmend aus dem Blick verliert. In grotesk-satirischer Verschärfung wird er durch das Festhalten an seinem Spezialinteresse, durch seine unaufhörliche,Zergliederung' und ,Isolation' (Košenina), selbst zu einer Kuriosität. Günter de Bruyn sah in Katzenberger „die verkörperte Deformierung des Menschen durch Arbeitsteilung“ und sogar „die Vorahnung unmenschlich gewordenen Spezialistentums" (de Bruyn 2004: 311), woraus er sehr weit führende Konsequenzen zog (ebd.). Jean Pauls Text selbst beurteilt bzw. löst das dargestellte Problem nicht, sondern verweist lediglich auf die ausschließlich am weltlichen Erkenntnisfortschritt und an ihren Forschungsobjekten interessierte Anatomie. Damit wird ein Raum für weiterführende Fragen eröffnet.

Auch die scheinbar unbeteiligte Haltung des Erzählers, der Katzenberger als einen Empiriker und nur durch seine äußeren Umstände zeigt, hat einen Doppelboden, wird der Leser doch dazu aufgefordert, sich mit dem Monstrum im eigenen Innern zu beschäftigen. Die oben zitierte technisch-medizinische Erklärung ,in jedem von uns [...] sind einige Ansätze zu einem Monstrum" (I/6, 198) muss auch metaphorisch verstanden werden, womit die Reduktion der 
Figur auf das rein Materielle erzähltechnisch entschärft wird. Am Ende wird Katzenbergers Verhältnis zu Menschen - es heißt nämlich: Er liebe sie nur so lange, wie lange er an ihnen lerne (I/6, 235) - als ,moralischer Leerdarm' beschrieben:

Katzenbergers Herz war in dieser Rücksicht vielleicht das Herz manches Genies; wenigstens so etwas von moralischem Leerdarm. Bekanntlich wird dieser immer in Leichen leer gefunden - nicht weil er weniger voll wird, sondern weil er schneller verdaut und fortschafft; - und so gibts Leer-Herzen, welche nichts haben, bloß weil sie nichts behalten, sondern alles zersetzt weitertreiben. (I/6, 235)

Katzenbergers ,moralischer Leerdarm' wird hier nicht als etwas Unmenschliches abgelehnt, sondern lediglich als eine Tatsache konstatiert. Umso effektvoller wirkt dabei die Metapher des ,Leer-Herzens', das zum leeren Darm einer Leiche in Analogie gesetzt wird. Dass im Inneren, im Herzen, nichts bleibt, ist die Folge der unaufhörlichen anatomischen und geistigen Sezierwut des Doktors. Die unerschöpfliche Fragmentierung bewirkt, dass auch das Herz alles zergliedert und nichts behält. Damit wird nicht nur die Vorstellung vom ,ganzen Menschen untergräbt, sondern auch eine Dimension des Menschlichen festgemacht, die in der Zeit der wachsenden Spezialisierung zum Vorschein kommt. Auch dem Hinweis darauf durfte der enthusiastische Dank des Anatomen Meckel gegolten haben.

\section{Literatur}

Bergengruen, Maximilian (2003): Missgeburten. Vivisektionen des Humors in Jean Pauls „Dr. Katzenbergers Badereise“. In: Jürgen Helm, Karin Stukenbrock (Hgg.): Anatomie. Sektionen einer medizinischen Wissenschaft im 18. Jahrbundert. Stuttgart: Franz Steiner, 271-292.

Bergengruen, Maximilian / Borgards, Roland / Lehmann, Johann Friedrich (2001): Einleitung. In: Dies. (Hgg.): Die Grenzen des Menschen. Anthropologie und Ästhetik um 1800. Würzburg: Königshausen \& Neumann, 7-14.

De Bruyn, Günter (2004): Das Leben des Jean Paul Friedrich Richter. Eine Biographie. 6. Aufl., Frankfurt a. M.: Fischer.

Dietrich, Ronald (2003): Der Gelehrte in der Literatur. Literarische Perspektiven zur Ausdifferenzierung des Wissenschaftssystems. Würzburg: Königshausen \& Neumann.

Häntzschel, Günter (2014): Sammel(l)ei(denschaft). Literarisches Sammeln im 19. Jahrhundert. Würzburg: Königshausen \& Neumann.

Helmreich, Christian (2002): "Einschiebeessen in meinen biographischen petits soupers". Jean Pauls Exkurse und ihre handschriftlichen Vorformen. In: Geneviève Espagne, Christian Helmreich (Hgg.): Schrift-und Schreibspiele. Jean Pauls Arbeit am Text. Würzburg: Königshausen \& Neumann, 99-122.

Jean Paul (2000): Dr. Katzenbergers Badereise nebst einer Auswahl verbesserter Werkchen. In: Ders.: Sämtliche Werke. Hg. von Norbert Miller. Abt. I, Bd. 6. Darmstadt: Wissenschaftliche Buchgesellschaft, 77-363.

Kaminski, Nicola (2017): „Nachdruck des Nachdrucks“ als Werk(chen)organisation oder wie D. Katzenberger die Kleinen Schriften von Jean Paul Friedrich Richter anatomiert. In: Jahrbuch der Jean Paul Gesellschaft (52), 29-70. 
Kemp, Friedhelm / Miller, Norbert / Philipp, Georg (Hgg.) (1963): Jean Paul. Werk, Leben, Wirkung. Texte. München: Piper \& Co., 7-24.

Košenina, Alexander (2004): Der gelehrte Narr. Gelehrtensatire seit der Aufklärung. Göttingen: Wallstein.

Meckel, Johann Friedrich (1815): De duplicitate monstrosa commentarius. Halle, Berlin: Waisenhausbuchhandlung.

Neumann, Peter Horst (1975): Die Werkchen als Werk. Zur Form- und Wirkungsgeschichte des Katzenberger-Korpus von Jean Paul. In: Jahrbuch der Jean Paul Gesellschaft (10), 151-186.

Noack, Bernd (2013): Vorliebe für groteske Missbildungen. In: Neue Zürcher Zeitung, v. 12.04.2013 [Zugriffsdatum: 05.07.2018].

Pfotenhauer, Helmut (2013): Jean Paul. Das Leben als Schreiben. Biographie. München: Carl Hanser.

Pott, Sandra (2002): Medizin, Medizinethik und schöne Literatur. Studien zu Säkularisierungsvorgängen vom 17. bis zum frühen 19. Jahrhundert. Berlin/New York: De Gruyter.

Schmitz-Emans, Monika (2016): Ut collectio poesis. Über das Sammeln als Kunst bei Jean Paul. In: Jahrbuch der Jean Paul Gesellschaft (51), 59-122.

Ueding, Gert (1993): Jean Paul. München: C. H. Beck.

Wieland, Magnus (2008): Teuflische Trinker: Satan, Sokrates, Sechsflaschenmann. In: Thomas Strässle, Simon Zumsteg (Hgg.): Trunkenheit. Kulturen des Rausches. Amsterdam/Atlanta: Rodopi, 153-173.

Wieland, Magnus (2011): Gestörter Organismus. Jean Pauls Ästhetik der Abweichung in der Erzählung „Dr. Katzenbergers Badereise“. In: New German Review. A Journal of Germanic Studies (24/1), $7-25$. 